\title{
Hamilton's new cellhost system for full automation of embryonic stem cell cultures
}

\author{
Stefanie Terstegge ${ }^{1}$, Jörg Pochert ${ }^{2} \&$ Oliver Brüstle ${ }^{1,3}$ \\ In pharmaceutical screening and cell biology research, the availability of cells can become a bottleneck in \\ the logistic chain of experiments. Hamilton and Life \& Brain have joined their expertise to develop a fully \\ automated system for the culture of primary cells, cell lines and embryonic stem (ES) cells, which provides \\ high-quality cells in large numbers.
}

\section{Importance of adherent cell cultures and stem cell cultures}

The demand for mammalian cells in pharmaceutical screening and cell biology research is constantly increasing. The evaluation of drug candidates is based on their biological effect on cell cultures in high-throughput screening while metabolic behavior and toxicity of new compounds are tested in cell-based adsorption, distribution, metabolism, elimination and toxicity (ADMET) assays. Basic research relies on numerous cell lines and primary cells, which constitute a tool for the elucidation of basic mechanisms of cell proliferation, differentiation and function. In this field, ES cells are gaining importance as a model system. In addition to their enormous differentiation potential, these cells possess the capacity for unlimited self-renewal, which facilitates their efficient genetic modification. Limitations of the current cell culture procedures include the lack of standardization associated with poor reproducibility and insufficient throughput.

\section{Challenges in automating ES cell cultures}

From a technical point of view, automated culture of ES cells constitutes a considerable challenge. One critical aspect is the phenomenon of uncontrolled differentiation in the delicate stem cell culture. Many of the ES cell culture systems still depend on coculture with fibroblasts. In terms of automation, this results in the demand for particularly gentle handling, including maintenance of a feeder cell layer during medium change. Adding to the challenge is the fact that many manual processes, such as hand movements for even distribution of the cells in the dish, are difficult to imitate using robots. Further issues concern general cell culture requirements: for example, avoiding contamination by bacteria and fungi, or standardizing the processes involved in preparing the cells for downstream experiments. As every automated device works on the principle of standardization and reduces the number of manual interventions,

\footnotetext{
${ }^{1}$ Life \& Brain GmbH, Cellomics Unit, Sigmund-Freud-Strasse 25, DE-53105 Bonn Germany. ${ }^{2}$ Hamilton Bonaduz AG, Via Crusch 8, CH-7402 Bonaduz, Switzerland. ${ }^{3}$ University of Bonn Medical Center, Institute of Reconstructive Neurobiology, Sigmund-Freud-Str. 25, DE-53105 Bonn, Germany. Correspondence should be addressed to J.P. (jpochert@hamilton.ch).
}

automation is bound to provide significantly better results compared to manual cell culture procedures. With cell ${ }^{\text {host }}$, Hamilton Bonaduz and Life \& Brain have developed a fully automated system for ES cell culture.

\section{Hamilton's fully automated cell ${ }^{\text {host }}$ system}

The cell ${ }^{\text {host }}$ system (Fig. 1) supports fully automated culture of ES cells in SBS-standard six-well microplates. It consists of a MICROLAB STAR pipetting robot, a sterile housing equipped with size filtration and two Kendro incubators, which can bring cell culture plates and medium tubs within the robot's operating range by using linear transfer units. The MICROLAB STAR features the

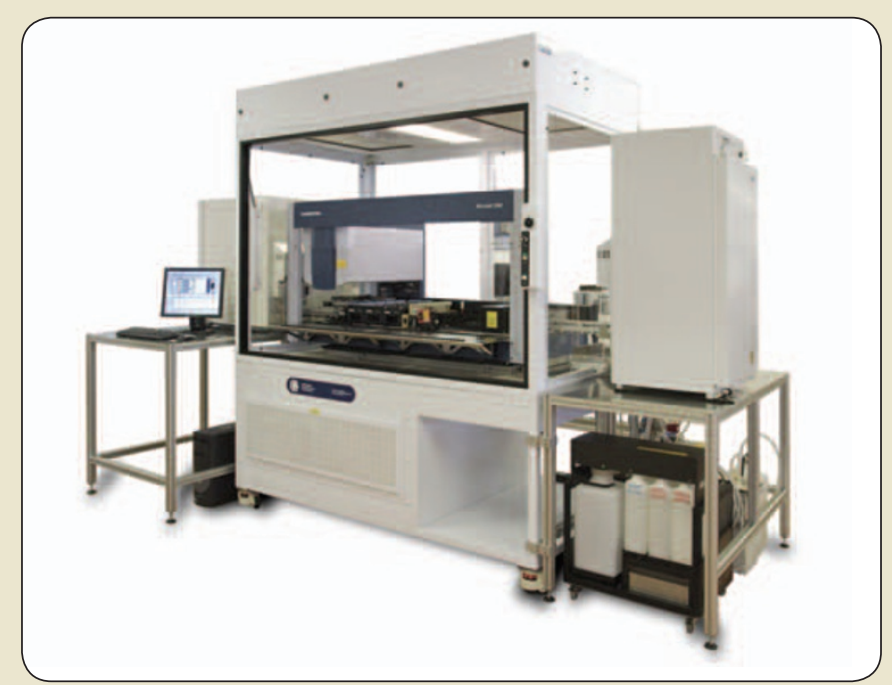

Figure 1 | cell host system. The core of the system is a Hamilton MICROLAB STAR pipetting robot. Cell cultures or cell culture media as well as growth factors are stored at different temperatures in Kendro incubators $\left(37^{\circ} \mathrm{C}\right.$ incubator for cell cultures; $4{ }^{\circ} \mathrm{C}$ incubator for media). Bar-code readers in each incubator guarantee safe and rapid access to the desired cell culture plates or medium containers. The working station is contained in a sterile housing with laminar airflow developed by Bigneat. 


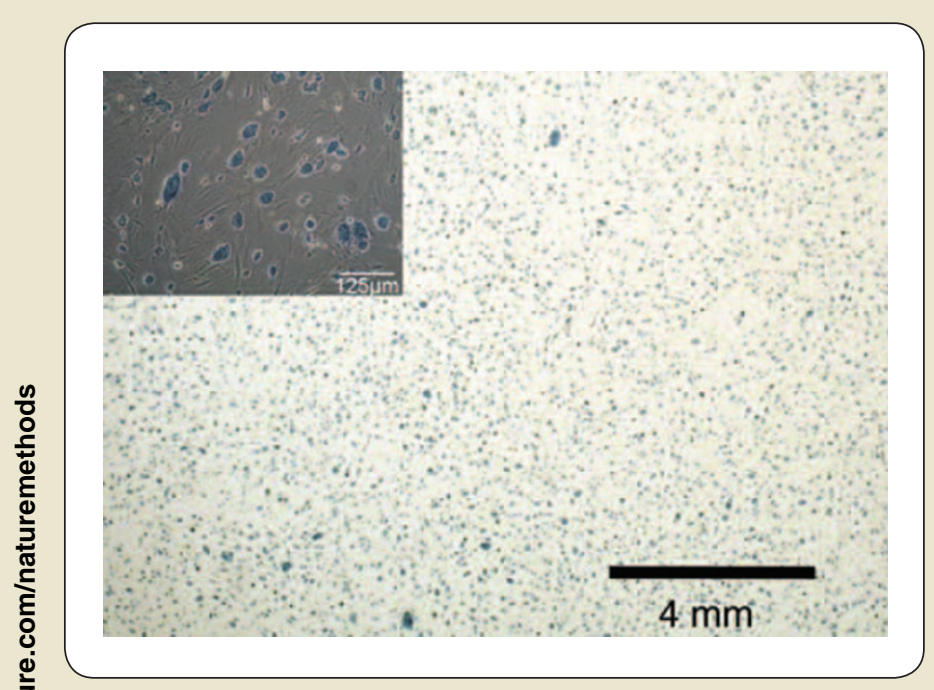

Figure 2 | Microscopic views of murine ES cells plated and incubated in the cell ${ }^{\text {host }}$ system. The inset shows a detail enlargement. The bar in the inset represents $125 \mu \mathrm{m}$. The cells were stained for alkaline phosphatase activity, a marker of pluripotency.

"monitored air displacement" pipetting principle, which functions in much the same way as a hand pipette. It operates without tubing, which means that pipetting of the medium and the cell suspensions does not involve system liquid, which can be an important source of contamination. Media are prepared in autoclavable aluminum containers and are brought to the right temperature immediately before use. Empty medium containers are promptly removed from the sterile environment.

Dedicated software package for data tracking and scheduling Any automated system devised for handling large numbers of plates, with each plate undergoing complex operations, requires sophisticated operating and data tracking capability. Hamilton's dedicated cell $^{\text {track }}$ software monitors and tracks every operation on every plate and enables data documentation in conformity with CFR 21 Part 11 requirements. The system software allows the selection of cell culture plates for particular processes, such as cell plating or addition of growth factors. With the cell ${ }^{\text {task }}$ scheduling software, groups of cell culture plates can be defined as input for iterative processes such as medium changes over long time periods. The cell ${ }^{\text {track }}$ database facilitates the comprehensive documentation of all process steps carried out. For every registered cell culture plate, a 'plate trail' lists all steps that the plate was subjected to.

\section{Automated plating of cells}

Plating with MICROLAB STAR leads to a homogenous distribution of murine ES cells on top of the feeder cell layer (Fig. 2). An even distribution of cells is particularly important when dealing with ES cells, as formation of cell clumps favors spontaneous differentiation. To achieve this result, the typical manual movements for distributing the cells across the plate are simulated by the robotic hand.



Figure 3 | Microscopic picture of a fibroblast layer immediately after a medium change with the MICROLAB STAR in the cell ${ }^{\text {host }}$ system (a) and after a less careful manual medium change (b). Each bar represents $250 \mu \mathrm{m}$. The robotic medium change leaves the cell layer undamaged.

\section{Automated medium change}

One of the most frequently required processes in plate handling is medium change. To minimize the residual volume, cell culture plates are raised at one side by means of a built-in automatic plate-lifter, and the medium is carefully aspirated at the lowest point of the well. The new medium is slowly dispensed at the edge of the well, thus avoiding disturbance of the cell layer. Microscopic investigation showed the intactness of the cell layer (Fig. 3a), in marked contrast to the results of a less cautious manual experiment (Fig. 3b).

\section{Cell growth}

ES cells propagated with cell ${ }^{\text {host }}$ retained their typical morphology after plating and medium change (Fig. 2, inset). The degree of spontaneous differentiation did not exceed the extent observed in manually treated cultures. Maintenance of pluripotent status was verified by histochemical detection of alkaline phosphatase activity. Cell viability after a culturing time of $48 \mathrm{~h}$ was comparable to that determined in the manual control experiment. No contamination was detectable.

\section{Conclusion}

The results of this study show the feasibility of automating ES cell culture. The system used here has the advantage of being based on manual methods and on being free of liquid-filled components. Cells easily survive various pipetting procedures such as mechanical plating and repeated changes of media. Microscopic observation shows that the typical morphology of ES cells is retained. Automated ES cell cultures show no increase in spontaneous differentiation, thus confirming stress-free and gentle handling of the cells by cellhost. Most importantly, the system operates in a contamination-free way. It is to be expected that the processes developed for the delicate culture of ES cells can be translated to less sensitive cell types commonly used in toxicological tests and pharmaceutical screening.

The company website is www.hamiltoncompany.com/product/ robotics/cellculture.html. For further information, please contact Hamilton at infoservice@hamiltonrobotics.com. 